\title{
Newborn with transient macular rash and thrombocytopenia
}

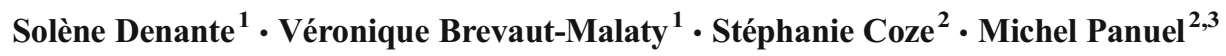

Published online: 17 June 2017

(C) ISS 2017

Keywords Infant $\cdot$ Newborn $\cdot$ Rubella $\cdot$ Bone

\begin{abstract}
Answer
Skeletal manifestations of congenital rubella syndrome
\end{abstract}

\section{Discussion}

Worldwide, congenital rubella syndrome (CRS) affects more than 100,000 infants each year $[1,2]$. The outcome of fetal infection is dependent on the gestational timing of maternal rubella, but fetal infection can occur at any stage of pregnancy. Fetal infection rates by the rubella virus (RV) are highest during the first trimester and during the last month of pregnancy. However, the teratogenic effects of RV leading to CRS only occur if the fetus is infected before the 18th week of pregnancy [3]. The only prevention is the vaccination, which had been introduced during the early 1970s and has led to a reduction in rubella and CRS cases of about $80 \%$ [2]. However, CRS can still be observed in developed countries. CRS can lead to

The case presentation can be found at doi: 10.1007/s00256-017-2693-7

Michel Panuel

michel.panuel@ap-hm.fr

1 Department of Neonatal Intensive Care Unit Hopital Nord Assistance Publique, Hôpitaux de Marseille, Marseille, France

2 Department of Medical Imaging Hôpital Nord Assistance Publique, Hôpitaux de Marseille, Marseille, France

3 Aix Marseille Université, CNRS, EFS, ADES UMR 7268, 13916 Marseille, France severe permanent manifestations, including sensorineural deafness, cataract, and cardiac (pulmonary valvular stenosis, pulmonary artery hypoplasia, and ventricular septal defect) and neurological (microcephaly) defects, in addition to transient abnormalities such as cutaneous, hematological, and skeletal changes [4]. The prenatal diagnosis of congenital rubella infection requires the positivity of maternal tests (RVIgM, RV-IgG, and/or detection of the virus by reverse transcription-polymerase chain reaction) and the detection of RV-IgM or viral genome in amniotic fluid or fetal blood [4, 5]. After birth, diagnosis is based on the same tests. There is no curative treatment; however, postnatal diagnosis is of major concern to provide an accurate follow-up plan. If the diagnosis has not been suspected during pregnancy, knowledge of the radiological signs may be helpful.

The radiographic features of osseous lesions due to intrauterine rubella infection consist of symmetrical metaphyseal alterations of the long bones [6, 7]. There are linear areas of increased bone density parallel to the longitudinal axis and intermingled with areas of radiolucency leading to the "celery stick" appearance (Figs. 2, 3). Lesions may also be limited to transversal or rounded radiolucent areas (Fig. 1); irregularity of the growth plate may be observed. The absence of periostitis is noted, spontaneous pathological fracture has been reported [8], and epiphyseal maturation of the knee is often delayed. Radiographic abnormalities result from inhibition of the mitotic activity of cartilaginous bone-forming cells by the rubella virus [6]. Complete recovery is usually and rapidly observed.

The same striated metaphyseal radiographic pattern is observed in newborns with cytomegalovirus infection; however, in this condition, bone maturation is normal [9]. Other transplacental infections such as Herpes virus, parvovirus B19 infections, or congenital syphilis may lead to metaphyseal changes but without a striated pattern [10]. 
In this reported case, the mother presented with rubella seroconversion between the 10th and 13th weeks of pregnancy. For the newborn, the macular rash disappeared 2 days after birth, the liver function tests and blood platelet count resolved quickly; there was no evidence of a cataract, but microcephaly was noted at birth. The child is now 3 years old. He has profound deafness requiring hearing aids complicated by severe language and behavioral disorders.

\section{Compliance with ethical standards}

Conflicts of interest The authors declare that they have no conflicts of interest.

\section{References}

1. Centers for Disease Control and Prevention. Progress toward control of rubella and prevention of congenital rubella syndromeworldwide, 2009. Morb Mortal Wkly Rep. 2010;59:1307-10.
2. Kancherlaa V, Oakley GP Jr, Brent RL. Urgent global opportunities to prevent birth defects. Semin Fetal Neonatal Med. 2014;19(3): 153-60.

3. Duszak RS. Congenital rubella syndrome: major review. Optometry. 2009;80:36-43.

4. Bouthry E, Picone O, Hamdi G, Grangeot-Keros L, Ayoubi JM, Vauloup-Fellous C. Rubella and pregnancy: diagnosis, management and outcomes. Prenat Diagn. 2014;34:1246-53.

5. Tang JW, Aarons E, Hesketh LM, et al. Prenatal diagnosis of congenital rubella infection in the second trimester of pregnancy. Prenat Diagn. 2003;23:509-12.

6. Reed GB. Rubella bone lesions. J Pediatr. 1969;74(2):208-13.

7. Rabinowitz JG, Wolf BS, Greenberg EI, Rausen AR. Osseous changes in rubella embryopathy. Radiology. 1965;85(3):494-500.

8. Sacks R, Habermann ET. Pathological fracture in congenital rubella. A case report. J Bone Joint Surg Am. 1977;59(4):557-9.

9. Kuhns LR, Slovis T, Hernandez R, Poznanski AK. Knee maturation as a differentiating sign between congenital rubella and cytomegalovirus infections. Pediatr Radiol. 1977;6:36-8.

10. Cantey JB, Pritchard MA, Sanchez PJ. Bone lesions in an infant with congenital parvovirus B19 infection. Pediatrics. 2013;131(5): e1659-63. 